\title{
Author Correction: Monitoring and long-term management of giant cell arteritis and polymyalgia rheumatica
}

Dario Camellino (D, Eric L. Matteson, Frank Buttgereit (D) and Christian Dejaco (D)

Nature Reviews Rheumatology (2020) https://doi.org/10.1038/s41584-020-0458-5 Published online 05 August 2020

In the originally published version of this article, an incorrect description of the Polymyalgia Rheumatica Activity Score (PMR-AS) was given. The sentence "This score is based on the patient's and physician's global assessment, morning stiffness, the ability of the patient to elevate the arms and CRP levels" has been corrected to "This score is based on the patient's pain assessment, physician's global assessment, morning stiffness, the ability of the patient to elevate the arms and CRP levels." This has been corrected in the HTML and PDF versions of the manuscript.

https://doi.org/10.1038/s41584-020-00505-x I Published online 10 September 2020

๑) Springer Nature Limited 2020 